\title{
How Immunotherapy Has Changed the Continuum of Care in Hepatocellular Carcinoma
}

\author{
Giulia Martini ${ }^{1}$, Davide Ciardiello ${ }^{1}$, Fernando Paragliola ${ }^{1}$, Valeria Nacca ${ }^{1}$, Walter Santaniello ${ }^{2}$, \\ Fabrizio Urraro ${ }^{3}{ }^{(D}$, Maria Stanzione ${ }^{4}$, Marco Niosi ${ }^{5}$, Marcello Dallio ${ }^{5}{ }^{\circ}$, Alessandro Federico ${ }^{5}\left({ }^{(}\right.$, \\ Francesco Selvaggi ${ }^{6}$, Carminia Maria Della Corte ${ }^{1}$, Stefania Napolitano ${ }^{1}$, Fortunato Ciardiello ${ }^{1}$ \\ and Erika Martinelli ${ }^{1, *}$
}

check for updates

Citation: Martini, G.; Ciardiello, D.; Paragliola, F.; Nacca, V.; Santaniello, W.; Urraro, F.; Stanzione, M.; Niosi, M.; Dallio, M.; Federico, A.; et al. How Immunotherapy Has Changed the Continuum of Care in Hepatocellular Carcinoma. Cancers 2021, 13, 4719. https://doi.org/ 10.3390/cancers13184719

Academic Editors: Lorenza Rimassa and Alfred Sze-Lok Cheng

Received: 4 August 2021

Accepted: 16 September 2021

Published: 21 September 2021

Publisher's Note: MDPI stays neutral with regard to jurisdictional claims in published maps and institutional affiliations.

Copyright: (c) 2021 by the authors. Licensee MDPI, Basel, Switzerland. This article is an open access article distributed under the terms and conditions of the Creative Commons Attribution (CC BY) license (https:/ / creativecommons.org/licenses/by/ $4.0 /)$.
1 Oncologia Medica, Dipartimento di Medicina di Precisione, Università degli Studi della Campania “L. Vanvitelli", 80100 Naples, Italy; giulia.martini@unicampania.it (G.M.); davide.ciardiello@unicampania.it (D.C.); fernando.paragliola@unicampania.it (F.P.); valeria.nacca@unicampania.it (V.N.); carminiamaria.dellacorte@unicampania.it (C.M.D.C.); stefania.napolitano@unicampania.it (S.N.); fortunato.ciardiello@unicampania.it (F.C.)

2 Chirurgia Epatobiliare e Trapianto di Fegato, A.O.R.N. Antonio Cardarelli, 80100 Naples, Italy; walter.santaniello@gmail.com

3 Radiologia, Dipartimento di Medicina di Precisione, Università degli Studi della Campania "L. Vanvitelli", 80100 Naples, Italy; fabrizio.urraro@policliniconapoli.it

4 Malattie Infettive, Dipartimento di Salute Mentale e Fisica e Medicina Preventiva, Università degli Studi della Campania “L. Vanvitelli", 80100 Naples, Italy; maria.stanzione@unicampania.it

5 Epato-Gastroenterologia, Dipartimento di Medicina di Precisione, Università degli Studi della Campania “L. Vanvitelli”, 80100 Naples, Italy; marco.niosi@policliniconapoli.it (M.N.); marcello.dallio@unicampania.it (M.D.); alessandro.federico@unicampania.it (A.F.)

6 Dipartimento di Scienze Mediche e Chirurgiche Avanzate, Università degli Studi della Campania “L. Vanvitelli”, 80100 Naples, Italy; francesco.selvaggi@unicampania.it

* Correspondence: erika.martinelli@unicampania.it

Simple Summary: HCC is a very aggressive disease and patients diagnosed in an advanced/metastatic setting obtain poor survival outcomes with standard treatments. In recent years, the introduction of immunotherapy strategies, such as immune checkpoint inhibitors as single agents and in combination with already approved local and systemic treatments, has strongly changed the therapeutic landscape of HCC. Soon, the discovery of novel potential immune targets, together with the understanding of potential biomarkers of resistance, will help to better define novel treatment opportunities for patients with HCC.

Abstract: Hepatocellular carcinoma (HCC) is one of the leading causes of death worldwide. The use of local treatment, such as surgical resection, liver transplant, and local ablation, has improved the survival of patients with HCC detected at an early stage. Until recently, the treatment of patients with metastatic disease was limited to the use of the multikinase inhibitor (MKI) sorafenib with a marginal effect on survival outcome. New target approaches, such as the oral MKI lenvatinib in first-line treatment and regorafenib, ramucirumab, and cabozantinib in later lines of therapy, have demonstrated efficacy in patients with preserved liver function (Child-Pugh class A) and good performance status. On the other hand, the implementation of immune checkpoint inhibitors directed against PD-1 (nivolumab and pembrolizumab), PD-L1 (atezolizumab), and anti-CTLA4 (ipilimumab) in the management of advanced HCC has strongly changed the continuum of care of HCC. Future research should include the evaluation of molecular biomarkers that can help patient selection and provide new insight on potential combined approaches. In this review, we provide an overview of the clinical evidence of the use of immune checkpoint inhibitors in HCC, and discuss how immunotherapy has been implemented into the continuum of HCC care.

Keywords: HCC; immune checkpoint inhibitors; multimodal treatment; biomarkers; AFP 


\section{Introduction}

Hepatocellular carcinoma (HCC) represents $90 \%$ of liver cancers and is one of the principal causes of death worldwide, with a steady rise of mortality rate [1]. Principal risk factors that might induce liver cirrhosis and are ultimately responsible for HCC disease are Hepatitis B or C viral chronic infections, alcohol abuse, obesity, diabetes, nonalcoholic steatohepatitis (NASH), nonalcoholic fatty liver disease (NAFLD), and certain inherited metabolic diseases [2]. HCC diverges from other solid tumors due to its origin in the context of cirrhosis. For this reason, a multidisciplinary approach with specialist figures, hepatologists, oncologists, surgeons, and radiologists, among others, is strongly required to guarantee correct patient management. HCC staging has been determined by different systems, with Barcelona Clinic Liver Cancer (BCLC) being the most common [3]. BCLC staging divides patients into stage $0 / A$ with very early/early disease, stage $B$ (intermediate) with multinodular HCC, stage $\mathrm{C}$ for advanced HCC amenable to systemic medical treatment, and stage $\mathrm{D}$ for patients with terminal disease only susceptible to palliative therapies [4]. Only patients included in stage 0/A are putative candidates for radical locoregional treatment modalities, such as surgical resection, radiofrequency ablation (RFA), and liver transplants, whereas transarterial chemoembolization (TACE) is reserved for stage B HCC [5]. Unfortunately, due to the lack of widespread screening programs that help detect the disease in early stages together with the stigma of risk factors (alcohol abuse, use of intravenous (IV) drugs) associated with the development of cirrhosis, HCC remains a silent killer. Since 2007, the mainstay of advanced HCC treatment has been limited to the use of a single drug, the oral multikinase inhibitor (MKI) sorafenib, with about three months increase in terms of survival and a series of related adverse events of importance [6]. Sorafenib is approved as a first-line strategy for patients with advanced disease, not amenable to locoregional treatments and transplant, with a Child-Pugh score of A or BCLC C criteria [7]. Recently, lenvatinib, another MKI, has received approval from the EMA and FDA and could be alternatively used in first-line settings, based on its non-inferiority activity compared to sorafenib [8]. Furthermore, in the field of targeted agents, regorafenib and cabozantinib failed to demonstrate non-inferiority to sorafenib in the first line but are approved for second-line treatment after sorafenib failure, as well as the anti-vascular endothelial growth factor receptor 2 (VEGFR2) antibody ramucirumab for patients with alpha-fetoprotein (AFP) $\geq 400 \mathrm{ng} / \mathrm{mL}$ [9-11].

In the last five years, the implementation of immunotherapy into the therapeutic armamentarium of HCC has strongly changed the medical approach to HCC patients. Therefore, immune checkpoint inhibitors (ICIs) have demonstrated promising results in Phase II and Phase III studies, and, to date, nivolumab and pembrolizumab have received accelerated approval by the Food and Drug administration (FDA) for second-line treatment of patients with advanced HCC after progression to sorafenib, while the combination of atezolizumab and bevacizumab has recently provided outstanding results in terms of overall survival (OS) and progression-free survival (PFS) in a first-line setting and has been recently approved by the FDA and European Medicines Agency (EMA) [12-14]. It is worth noting that several studies are currently investigating the combination of ICIs with different drugs, including targeted agents and chemotherapy, and novel immunotherapy strategies beyond ICIs are in current development. We herein provide an overview of the immune landscape of HCC, discuss the results of principal clinical trials that have led to currently approved immunotherapies and investigate potential immune biomarkers of response to improve outcomes in a disease that has always needed efficacious treatments.

\section{Immune Landscape of Hepatocarcinoma Disease}

The liver has a peculiar anatomy and fulfills functions including uptake of arterial and portal blood, filtration of gut pathogens, and excretion of toxic waste materials, a feature that leads to exposure to a high load of antigens [15]. This hepatic reticulo-endothelial system that comprises sinusoids, Kuppfer cells, and liver endothelial sinusoidal cells (LESC) activates innate T-cells through antigen presentation, causing a tolerogenic immune 
response in a physiological status [16]. It has been reported in several studies how the immune surveillance system could be damaged in the context of cirrhosis. This continuous inflamed status of the liver leads to the recruitment of cytokines and immune components, ultimate actors in neoplastic dysregulation in which HBV and HCV viruses could contribute to carcinogenesis development [17]. Immune tolerance that characterizes the evolution of HCC is regulated by innate and adaptive immune cells present in the immune tumor microenvironment (TME) such as CD4+ and CD8+ T-cells, dendritic cells (DCs), natural killer (NK) cells, myeloid-derived suppressor cells (MDSC), tumor-associated macrophages (TAMs) that express and up-regulate immune checkpoints on their surface as programmed cell death protein 1 (PD-1) and the cytotoxic lymphocyte protein 4 (CTLA-4) [18]. PD-1 is responsible for T-cell exhaustion and prevents T-cell activation by releasing cytotoxic mediators, and CTLA-4 impedes activation of T-cells by replacing CD28 in the interaction with CD80/86 ligands on antigen-presenting cells (APC) [19].

All these components of TME act with intricate processes as decreased tumor-associated antigen (TTA) recognition, accumulation of immune suppressive cells, and interaction between immune checkpoints and their ligands, leading to a final balanced immunotolerant status [20]. Moreover, the immunotolerance of liver cancer is accompanied by the release of several cytokines and regulatory factors, such as transforming growth factor (TGF)- $\beta$, which acts as an immunosuppressive factor [21].

\section{Clinical Evidence of Immune Checkpoint Inhibitors in Hepatocarcinoma}

Antigen presentation by major histocompatibility complex (MHC) is required for T-cell priming [22]. However, a cooperation between checkpoint molecules such as PD-1, PD-L1, CTLA-4, TIM-3, and LAG3 on T-cells and their ligands on antigen-presenting cells (APC) with the help of co-stimulatory molecules on receptors and ligands is needed for the activation of T-cells and finally establish a suppression of immune response against external antigens [23]. In a cirrhotic liver, the overexpression of suppressive checkpoint molecules with low production of cytokines in TME leads to a phenomenon called T-cell exhaustion, characterized by low immune activity and defective action against cancer [24]. Furthermore, the presence of a great number of antigens in liver TME complicates T-cell exhaustion. Hence, the use of ICIs has been explored in HCC to restore T-cell exhaustion and block HCC progression [25].

\subsection{Nivolumab}

The anti-PD-1 antibody nivolumab was investigated in the CheckMate 040 Phase I/II trial in 262 patients with advanced HCC, the majority (70\%) after sorafenib failure. Response rate (RR) was $20 \%$ in the expansion cohort of the study and this result led to approval by the FDA for second-line treatment of HCC. The expansion phase of this study included a combination cohort in which patients received nivolumab alone or in combination with ipilimumab (anti-CTLA-4). In particular, patients were randomized 1:1:1 to receive nivolumab $1 \mathrm{mg} / \mathrm{kg}+$ ipilimumab at $3 \mathrm{mg} / \mathrm{kg}$ administered every 3 weeks, followed by nivolumab $240 \mathrm{mg}$ every 2 weeks (Arm A), nivolumab $3 \mathrm{mg} / \mathrm{kg}+$ ipilimumab $1 \mathrm{mg} / \mathrm{kg}$ administered every 3 weeks, followed by nivolumab $240 \mathrm{mg}$ every 2 weeks (Arm B) or nivolumab $3 \mathrm{mg} / \mathrm{kg}$ every 2 weeks + ipilimumab $1 \mathrm{mg} / \mathrm{kg}$ administered every 6 weeks (Arm C). Arm A (3 mg/kg every 3 weeks (4 doses), followed by nivolumab $240 \mathrm{mg}$ every 2 weeks) reported the best results in terms of RR and overall survival rate at 12, 24, and 36 months of 61,48 , and $44 \%$, respectively, and the combination obtained accelerated approval by the FDA [12].

By contrast, the phase III randomized CheckMate 459 trial (NCT02576509) which explored the efficacy and safety of nivolumab treatment in first-line treatment compared with sorafenib standard treatment did not show a statistically significant improvement in the primary endpoint of the overall survival (OS) (16.4 months versus 14.7 months in the nivolumab and sorafenib arm, respectively, Hazard Ratio (HR): 0.85; Confidence Interval (CI) $0.72-1.02 ; p=0.0752$ ), and in median progression-free survival (mPFS) (3.7 months 
vs. 3.8 months). However, RR was significantly higher in the experimental arm (15\%) compared to $7 \%$ in the sorafenib control arm [26]. Although nivolumab treatment showed a clinical benefit in all the preplanned patient subgroups, with a favorable toxicity profile, the study did not change the standard of treatment for patients with naïve advanced HCC.

\subsection{Pembrolizumab}

Staying on the theme of anti-PD-1 ICIs, the KEYNOTE-224 Phase II trial reported data about the use of pembrolizumab after sorafenib failure. A RR of $17 \%$ and median OS of 12.9 months led to FDA accelerated approval of pembrolizumab in second-line settings [13]. However, results coming from the confirmatory randomized Phase III KEYNOTE240 trial failed to meet the prespecified co-primary endpoints of the trial OS and PFS with pembrolizumab compared with placebo in previously treated patients, although a survival improvement was obtained with respectively $16.9 \%, 13.9$ months, and 3.0 months RR, OS, and PFS in the experimental arm, compared to $4.4 \%, 10.6$ months, and 2.8 months in the control arm [27]. Even if not statistically significant, these data confirm the favorable risk-to-benefit ratio of the use of pembrolizumab after sorafenib failure, as compared with best supportive care.

\subsection{Atezolizumab}

The anti-Programmed Death-Ligand 1 (PD-L1) antibody atezolizumab was explored in a first-line setting in the Phase Ib GO30140 trial (NCT02715531) alone or in combination with the anti-VEGF monoclonal antibody bevacizumab. Arm A of the trial evaluated the combination of atezolizumab and bevacizumab every three weeks whereas Arm F randomized patients 1:1 to receive atezolizumab plus bevacizumab or atezolizumab as a single agent. The primary endpoint of Arm A, RR, was 36\%, while PFS, the primary endpoint of Arm F, was 5.6 months with the combination and 3.4 months with atezolizumab single agent (HR: $0.55 ; 80 \%$ CI $0.4-0.74 ; p=0.0108)$. Regarding safety profile, which was another co-primary endpoint of the study, a higher rate $(68 \%)$ of treatment-related adverse events (TRAEs) was reported in atezolizumab plus bevacizumab combination versus $41 \%$ in the atezolizumab single-agent arm [28]. Subsequently, the Phase III Imbrave150 trial randomized 2:1 501 patients to receive a combination of atezolizumab plus bevacizumab or sorafenib as first-line treatment, respectively. The primary endpoints of the study were OS and PFS as per the independent review facility (IRF) assessed-response evaluation criteria in solid tumors (RECIST) 1.1.

Therefore, median OS was not reached in the atezolizumab plus bevacizumab combination that resulted in 13.2 months in the sorafenib group (HR: 0.58; 95\% CI 0.42-0.79; $p=0.0006)$. OS rates were $84.8 \%$ and $67.2 \%$ with the experimental combination and the sorafenib arm obtained $72.2 \%$ and $54.6 \%$ at 6 and 12 months, respectively (HR: 0.58; 95\% CI $0.42-0.79 ; p=0.0006)$. Median PFS was 6.8 months in the combination arm and 4.3 in the sorafenib arm (HR: $0.59 ; 95 \%$ CI $0.47-0.76 ; p<0.0001$ ). The RR was $28 \%$ in the experimental arm versus $12 \%$ with control (95\% CI $23-33$ vs. $7-17$, respectively, $p<0.0001$ ), but reached $33 \%$ versus $13 \%$ (95\% CI $28-39$ vs. 95\% CI 8-19; $p<0.0001)$ when evaluated per modified RECIST (mRECIST) criteria. Toxicities reported were more frequent with the experimental combination, with hypertension the most common grade 3-4 TRAE [14]. These data changed clinical practice with breakthrough approval of atezolizumab plus bevacizumab combination by the FDA and EMA as first-line treatment of patients with unresectable or metastatic HCC who had not received systemic therapy. In the same way, the updated OS analysis conducted with 12 months of additional follow-up from primary analysis confirmed the strong efficacy of atezolizumab + bevacizumab combination over sorafenib treatment, with median overall survival of 19.2 months for the combination versus 13.4 months for sorafenib. Moreover, updated results of RR were consistent with the ones from the primary analysis $(29.8 \%$ per RECIST 1.1$)$ with a higher rate of complete response $(7.7 \%)$ than previously reported [29]. 


\subsection{Anti-CTLA-4 Plus Anti-PD-1/PD-L1 Antibodies Combinations}

The CTLA-4 immune receptor, expressed primarily on T regs and active $\mathrm{T}$ lymphocytes, binds CD80 and CD86 ligands, avoiding their interaction with CD28 thus limiting the antigen presentation process mediated by CD28, and dampening immune response [30]. The inhibition of CTLA-4 has been extensively studied in different solid tumors, such as melanoma and lung cancer [31,32]. Regarding HCC, the anti-CTLA-4 inhibitor tremelimumab has been explored as a single treatment or in combination with the anti-PD-L1 durvalumab as a second-line treatment strategy. In addition, a tremelimumab single agent has been investigated in patients with chronic HCV infection, in a Phase II clinical trial (NCT01008358), showing promising data with $17.6 \%$ RR, 76.4 disease control rate (DCR) and 6.48 months in terms of time to progression (TTP) [33]. After that, tremelimumab was investigated in combination with durvalumab in a randomized Phase II trial (NCT02519348) that enrolled 332 patients after sorafenib treatment failure, randomly assigned to the combination of tremelimumab at $300 \mathrm{mg}$ + durvalumab $1500 \mathrm{mg}$, durvalumab single agent, tremelimumab single agent or tremelimumab at $75 \mathrm{mg}+$ durvalumab. Safety, the primary endpoint of the study, was acceptable across all four arms, with the most common TRAE alanine aminotransferase (ALT) and aspartate aminotransferase (AST) increase, lipase increase, amylase increase, and diarrhea. Grade $\geq 3$ TRAE in the 4 arms occurred in $37.8 \%$, $20.8 \%, 43.5 \%$, and $24.4 \%$, respectively. RR by RECIST 1.1 and OS were secondary endpoints. Confirmed RRs (95\% CI) were $24.0 \%$ (14.9 to 35.3 ), $10.6 \%$ (5.4 to 18.1 ), $7.2 \%$ (2.4 to 16.1 ), and $9.5 \%$ (4.2 to 17.9), respectively. OS was higher in tremelimumab $300 \mathrm{mg}$ combined with the durvalumab arm with 18.7 (10.78 to 27.27) months, followed by tremelimumab single agent (15.11 (11.33 to 20.50) months), durvalumab single agent (13.57 (8.74 to 17.64) months), and tremelimumab $75 \mathrm{mg}+$ durvalumab arm (11.30 (8.38 to 14.95) months) [34,35].

The efficacy and safety findings coming from the combination arm with tremelimumab at $300 \mathrm{mg}$ have opened an avenue to the ongoing Phase III HIMALAYA trial (NCT03298451) of tremelimumab + durvalumab treatment compared to sorafenib in a first-line setting, where results are strongly awaited, as the two drugs were already granted orphan drug designation by the FDA in January 2020 [36].

\section{Combining Immune Checkpoint Inhibitors with Targeted Agents}

The development of HCC structure from dysplasia is a process influenced by proangiogenic factors such as angiopoietins, VEGF, transforming growth factors, basic fibroblast growth factors (bFGF), and platelet-derived growth factor (PDGF) that are secreted by TME and enhance tumor blood requirement from arteries to guarantee growth and metastatic spread. By contrast, tumor vessels are structurally imperfect and with a network of sinusoids that differ from hepatic sinusoids of a normal liver because they are more "capillarized" for the presence of a basement membrane and lack of fenestration [37]. Based on that, anti-VEGF strategies have represented a mainstay in HCC treatment for many years.

The MKI sorafenib inhibits tumor angiogenesis and tumor-cell proliferation and was approved in 2007 for patients with advanced HCC, medical treatment naïve, with ChildPugh A disease, after the results of the multicentric Phase III randomized SHARP clinical trial (NCT00105443) that enrolled 602 patients. Sorafenib treatment obtained an advantage in terms of OS compared to placebo group (10.7 months vs. 7.9 months; HR: 0.69; 95\% CI: $0.55-0.87 ; p<0.001$ ) with survival rates at 1 year of $44 \%$ in the sorafenib group and $33 \%$ in the placebo arm [6]. Another chance for first-line treatment for advanced HCC is the MKI lenvatinib, which obtained FDA and EMA approval after the results of non-inferiority Phase III REFLECT trial (NCT01761266) for patients with absence of portal vein invasion, clear bile duct invasion, and 50\% liver volume occupancy. Lenvatinib was not inferior to sorafenib treatment and had a tolerable safety profile [8].

Since then, treatment with other MKI molecules regorafenib and cabozantinib has been explored, without achieving statistically significant benefit in first-line treatment but in second-line treatment in the RESORCE (NCT01774344) and CELESTIAL (NCT01908426) Phase III trials, respectively. Interestingly, regorafenib treatment provided a median OS 
of 10.6 months, while the median OS of the sequence represented by sorafenib in firstline treatment and regorafenib in second-line treatment was 26.0 months, compared to 19.2 months with sorafenib and then placebo [9]. On the other hand, in the CELESTIAL trial, cabozantinib treatment in second or subsequent lines provided 10.2 months OS compared to 8 months with placebo (HR: $0.76 ; 95 \%$ CI $0.63-0.92 ; p=0.005$ ) and 5.2 months median PFS with cabozantinib and 1.9 months with placebo (HR: $0.44 ; 95 \%$ CI, $0.36-0.52$; $p<0.001$ ) [10].

Furthermore, the inhibition of angiogenesis in HCC was investigated with the anti-VEGF receptor 2 (VEGFR2) monoclonal antibody ramucirumab in the REACH trial (NCT01140347) and in the REACH-2 trial (NCT02435433). The REACH trial did not achieve statistically significant results but the analysis of a subgroup of "biomarker selected" patients with AFP $\geq 400 \mathrm{ng} / \mathrm{mL}$ showed a significant benefit in terms of OS (7.8 months in the ramucirumab arm compared to 4.2 months with placebo, HR: $0.67,95 \%$ CI $0.51-0.90$ ); based on that, the REACH-2 trial only included patients with AFP $\geq 400 \mathrm{ng} / \mathrm{mL}$, obtaining significant OS advantage, confirming the previous trial analysis [11,38]. These data have opened the door to putative sequence strategies with MKI inhibitors in advanced HCC, as described in Figure 1.

FIRST LINE

SECOND LINE

THIRD LINE

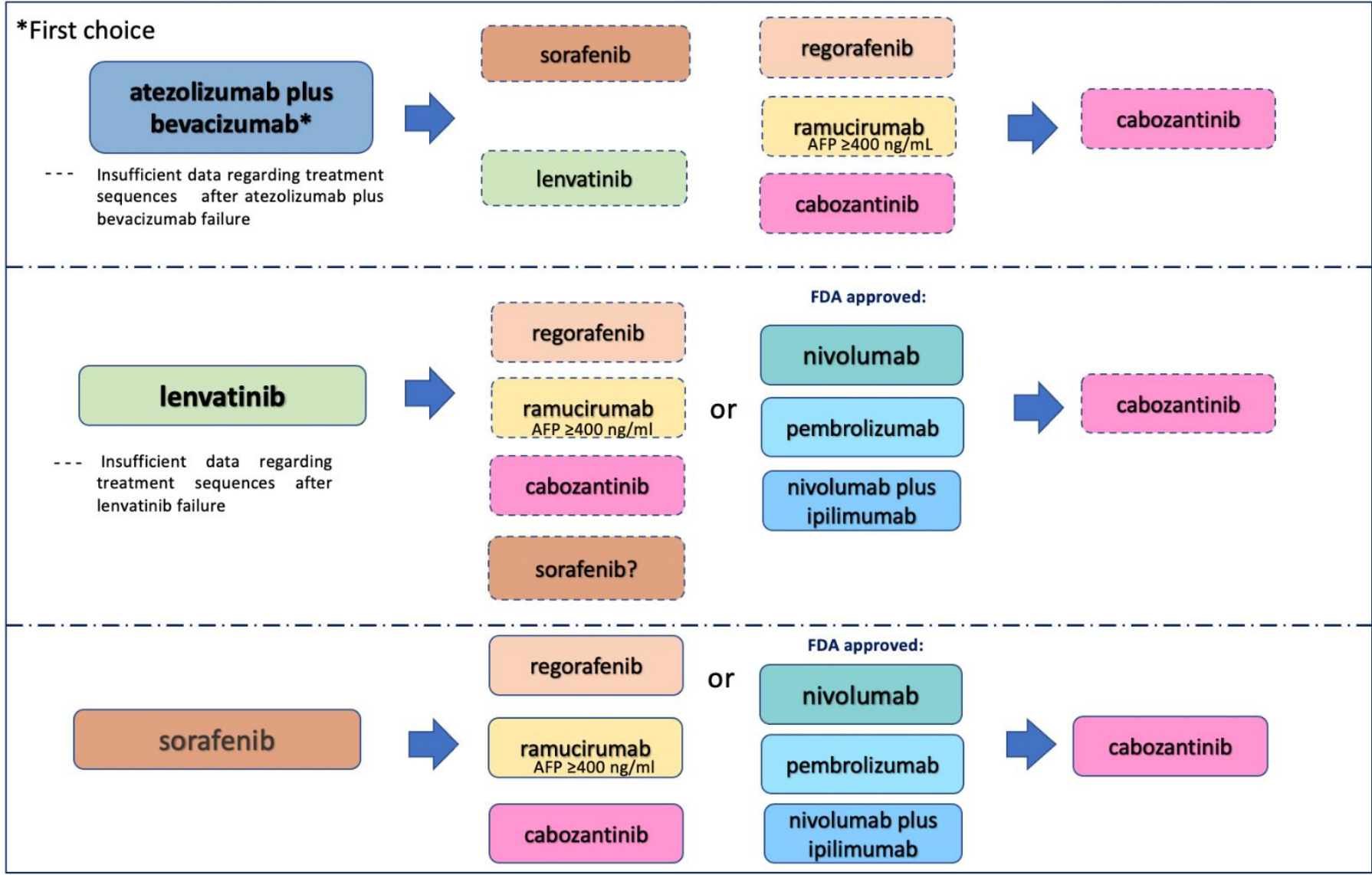

Figure 1. Different treatment scenarios in hepatocellular carcinoma.

Importantly, the tumor vessel-altered phenotype not only contributes to the new angiogenesis process that nourishes liver tumor but also determines a down-regulation of immune TME effectors such as CD8+ T-cells and a final immunosuppressive microenvironment in which $\mathrm{T}$ regs, MDSCs, and M2 polarized TAMs release immunosuppressive cytokines and finally block T-cells, NK cells, and DCs activation [39].

Based on this strong rationale, the combination of immune checkpoint inhibitors with anti-angiogenic drugs was examined in HCC and has proved effective strategies as per 
the case of the IMBRAVE150 trial of atezolizumab plus bevacizumab mentioned above. Furthermore, sintilimab plus anti-VEGF monoclonal antibody IBI305 safety and efficacy is currently being explored in first-line settings in the ongoing Phase II/III ORIENT-32 trial (NCT03794440). The same combination approach has been tried in second-line treatment with the anti-VEGFR-2 ramucirumab plus anti-PD-L1 durvalumab in the HCC cohort of the NCT02572687 Phase Ib trial. RR was $11 \%$ in the entire cohort but increased to $18 \%$ in the PD-L1 positive selected population, with a globally safe profile, suggesting future possible larger investigations in this setting [40].

Combining an anti-PD1/PD-L1 inhibitor to MKIs could represent another possible strategy under current development into different clinical trials (Table 1). Of note, lenvatinib in association with pembrolizumab as the first-line therapy determined in the KEYNOTE524 Phase Ib trial (NCT03006926) a $46 \%$ RR per mRECIST (95\% CI 36.0-56.3\%) and $36 \%$ per 1.1 RECIST (95\% CI $26.6-46.2 \%$ ), with a median duration of response (DoR) of 8.6 months (95\% CI 6.9 months to not estimable [NE]) per mRECIST and 12.6 months (95\% CI 6.9 months-NE) per RECIST v1.1, median PFS 9.3 months per mRECIST and 8.6 months per RECIST v1.1 and median OS 22 months. Grade $\geq 3$ TRAE occurred in $67 \%$ of patients; hypertension $(36 \%)$, diarrhea $(35 \%)$, and fatigue $(30 \%)$ were the most common [41]. Similarly, the ongoing randomized Phase III LEAP-002 trial (NCT03713593) explores the efficacy of lenvatinib alone or in combination with pembrolizumab in patients with Child-Pugh A advanced HCC. Based on that, other promising data come from the Camrelizumab in Combination with Apatinib in Patients with Advanced Hepatocellular Carcinoma (RESCUE) nonrandomized, Open-label, Phase II trial. Seventy patients were treated with a combination of camrelizumab + apatinib in first-line and 120 in second-line settings. RR was $34.3,95 \%$ CI (23.3 to 46.6 ) and $22.5 \%$, (15.4 to 31), mPFS was 5.7 (95\% CI, 5.4 to 7.4$)$ and 5.5 months ( $95 \%$ CI, 3.7 to 5.6$)$, and 12-month survival rate was $74.7 \%$ (95\% CI, 62.5 to 83.5$)$ and $68.2 \%$ (95\% CI, 59.0 to 75.7$)$, in first-line and second-line cohorts, respectively. Updated results with long-term follow-up recently presented by the authors showed remarkable data in terms of mOS 20.1 months for first-line treatment ( $95 \% \mathrm{CI}, 14.9$ to NE) and 21.8 months for second-line treatment (95\% CI, 17.3 to 26.8), demonstrating promising efficacy with manageable toxicity profile (hypertension being the most common TRAE experienced by $74 \%$ of patients) [42,43]. Moreover, cabozantinib has been investigated in combination with nivolumab/nivolumab + ipilimumab in a cohort of the CheckMate 040 trial (NCT01658878) obtaining clinically meaningfully results in terms of RR (17\% and $26 \%), \mathrm{DCR}(81 \%$ and $83 \%$ ), and mPFS (5.5 and 6.8 months) for doublet and triplet treatment, respectively. Although a high number of TRAE was observed in the triplet arm over the doublet arm, all the events were manageable. Furthermore, the COSMIC312 Phase III trial (NCT03755791) is currently investigating the efficacy of cabozantinib + atezolizumab compared with sorafenib in first-line settings. Final results are strongly awaited since preliminary data regarding the primary endpoint $\mathrm{mPFS}$ have shown a significant reduction in the risk of disease progression or death by $37 \%$ with the combination of cabozantinib + atezolizumab.

Other current studies are testing MKI association with immunotherapy as sorafenib in combination with nivolumab or pembrolizumab or regorafenib in combination with pembrolizumab in the NCT03347292 trial and avelumab in the REGOMUNE trial, respectively, and cabozantinib in combination with nivolumab/nivolumab plus ipilimumab in a cohort of the CheckMate 040 trial (NCT01658878) (Table 1). Another attractive strategy has investigated the use of chemotherapy in combination with ICIs for unresectable/metastatic HCC as the case of a Phase II trial testing camrelizumab plus FOLFOX-4)5-fluorouracil plus oxaliplatin) or GEMOX (gemcitabine plus oxaliplatin) chemotherapy regimens in advanced HCC or biliary tract cancer. In the HCC cohort RR was $26.5 \%$, disease control rate (DCR) $79.4 \%$ and mPFS 5.5 months. Additionally, camrelizumab in combination with FOLFOX is currently being investigated in the Phase III NCT03605706 trial (Table 1). 
Table 1. Principal ongoing clinical trials with immune checkpoint inhibitors in advanced HCC.

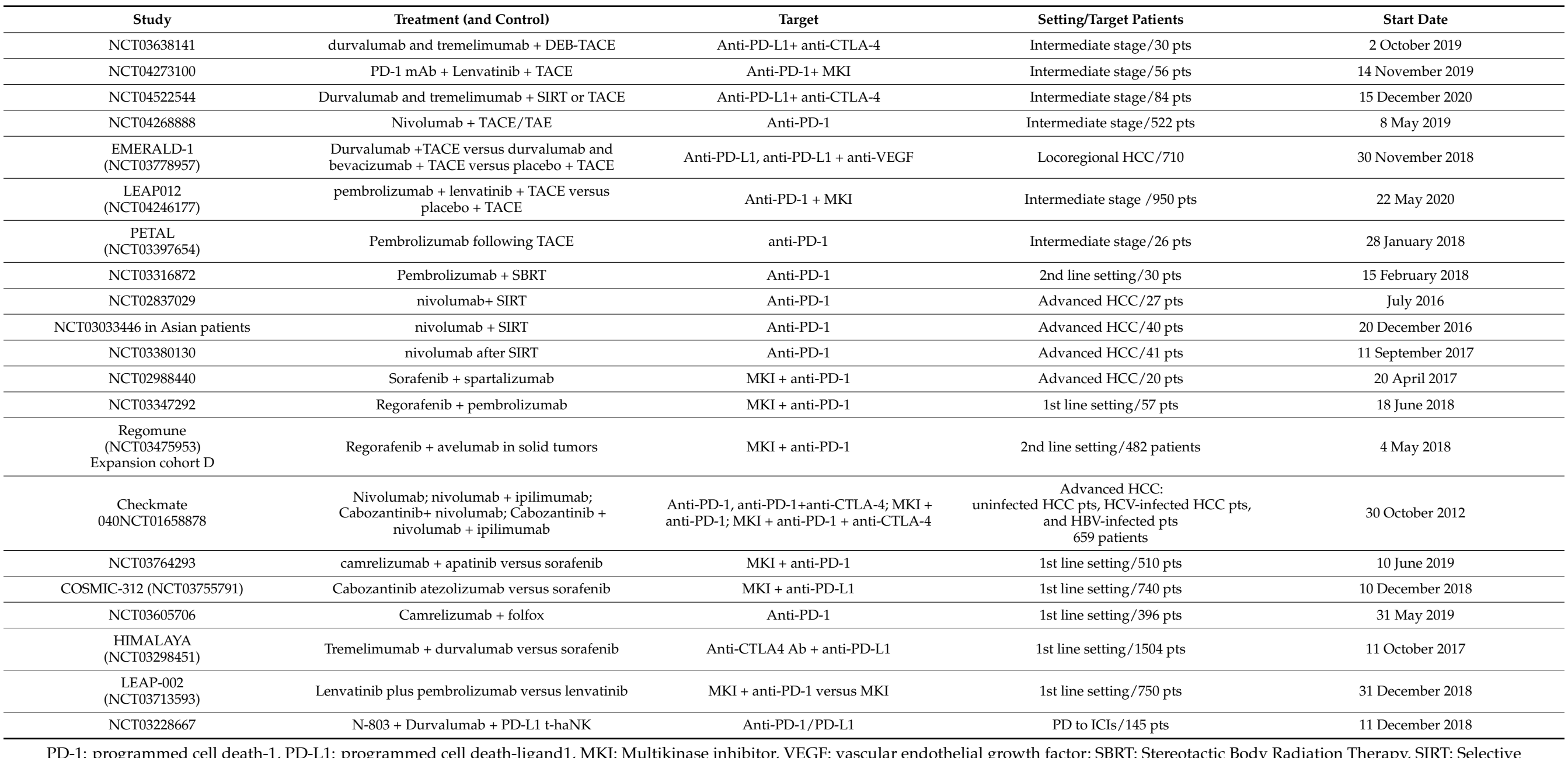

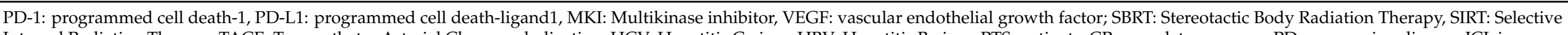

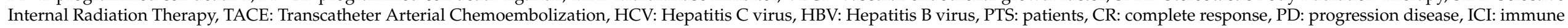
checkpoint inhibitor. 


\section{Same Approach in a Different Setting}

HCC has few chances of curability in advanced settings. Regarding the early stage defined by BCLC 0 or A criteria, different local approaches have provided an advantage in terms of efficacy and relapse-free survival, while chemotherapy or targeted agents have failed to demonstrate efficacy. In recent years, scientists have tried to harness the benefits of widely used locoregional approaches in combination with novel immune strategies. Giving the inflamed nature of liver tumor determined by its immunosuppressive phenotype, ICIs could prevent immune escape and avoid local recurrences after surgery. To this extent, anti-PD-1 and PD-L1 monoclonal antibodies are under investigation in adjuvant settings (Table 1). It is worth noting that the EMERALD-2 Phase III randomized trial (NCT03847428) is being conducted in patients with high risk of recurrence after surgery to study if durvalumab alone or in combination with bevacizumab provides better results compared to placebo in the adjuvant setting (Table 2).

Table 2. Principal ongoing clinical trials with immune checkpoint inhibitors in adjuvant HCC.

\begin{tabular}{|c|c|c|c|c|}
\hline Study & Treatment (and Control) & Target & Setting/Target Patients & Start Date \\
\hline $\begin{array}{l}\text { EMERALD-2 } \\
\text { (NCT03847428) }\end{array}$ & $\begin{array}{c}\text { Durvalumab versus } \\
\text { durvalumab + bevacizumab } \\
\text { versus placebo }\end{array}$ & Anti-PD-L1 + anti-VEGF & Adjuvant setting/888 pts & 29 April 2019 \\
\hline $\begin{array}{c}\text { Keynote } 937 \\
\text { (NCT03867084) }\end{array}$ & $\begin{array}{l}\text { Pembrolizumab } \\
\text { Versus placebo }\end{array}$ & Anti-PD-1 & $\begin{array}{c}\text { Adjuvant setting/ } \\
\text { CR after surgery or local } \\
\text { ablation/950 pts }\end{array}$ & 28 May 2019 \\
\hline $\begin{array}{l}\text { Checkmate 9DX } \\
\text { (NCT03383458) }\end{array}$ & Nivolumab versus placebo & Anti-PD-1 & $\begin{array}{c}\text { Adjuvant setting/ } \\
\text { CR after surgery or local } \\
\text { ablation } / 530 \text { pts }\end{array}$ & 18 April 2018 \\
\hline $\begin{array}{c}\text { IMbrave050 } \\
\text { (NCT04102098) }\end{array}$ & $\begin{array}{l}\text { Atezolizumab + bevacizumab } \\
\text { versus active surveillance }\end{array}$ & Anti-PD-L1 + anti-VEGF & Adjuvant setting/662 pts & 31 December 2019 \\
\hline $\begin{array}{c}\text { Jupiter04 } \\
\text { (NCT03859128) }\end{array}$ & Toripalimab versus placebo & Anti-PD-1 & $\begin{array}{c}\text { Adjuvant setting/ } \\
\text { CR after surgery/402 pts }\end{array}$ & 1 March 2019 \\
\hline
\end{tabular}

\section{Immunotherapy in Combination with Locoregional Treatment}

As locoregional treatments represent the mainstay of HCC treatment, different strategies have explored their association with immunotherapy in early-stage disease settings. For example, TACE treatment reserved for patients with BCLC B HCC has been investigated in combination with sorafenib in the TACTIS trial, providing PFS of 25.2 months compared to 13.5 months with TACE alone $p=0.006$ ) but unfortunately this advantage was not confirmed in terms of OS [44]. Then, immunotherapy has been associated with TACE treatment, based on the assumption of tumor necrosis induced by high intra-tumoral temperature that leads to the high release of TTAs and activation of liver-immune TME. Thus, several "ICI plus TACE" strategies are in progress and encouraging results from preliminary safety analyses justify further scientific development [45]. Moreover, novel studies are exploring if the TACE tissue damage could enhance the efficacy of anti-VEGF treatments as the EMERALD-1 trial (NCT03778957) of durvalumab and bevacizumab plus TACE, while the Phase III LEAP-012 is currently investigating pembrolizumab plus lenvatinib combination with TACE in incurable/non-metastatic HCC (NCT04246177).

Regarding other locoregional treatments, transarterial selective internal radiation therapy (SIRT) with Yttrium 90 microspheres has been investigated in BCLC B HCC as an alternative to TACE and current novel strategies are exploring its use in combination with ICIs, as pembrolizumab plus SIRT (NCT03033446), nivolumab plus SIRT (NCT02837029) in Asian patients and nivolumab after SIRT (NCT03380130). Furthermore, stereotactic body radiotherapy (SBRT) in association with pembrolizumab is being testing in the Phase II NCT03316872 study, after sorafenib failure. 


\section{Strategies beyond ICI}

Alternative immune strategies apart from ICIs in HCC are in current development. HCC disease is characterized by a high presence of TAAs, hence the use of vaccines that target TAAs being investigated. Principal targets of vaccine therapy in HCC have been AFP, glypican-3 (GPC-3), MAGE-1, human telomerase reverse transcriptase (hTERT), and NY-ESO-1. Interestingly, in a study that included $41 \mathrm{HCC}$ and 24 non-HCC patients, the authors identified the capacity of circulating T-cells to recognize AFP-derived CD4+ T-cell epitopes and how anti-AFP CD4+ T-cell response was evident only in HCC subgroups with mild or elevated AFP serum levels, suggesting the potential use of anti-AFP cancer vaccines in HCC [46]. However, preliminary results coming from other analyses have provided mixed data, due to the small number of cases analyzed, deficient CD4+ helper T-cell support, or low numbers of antigens used in the vaccine. It is worth noting that encouraging results in terms of survival benefit have come from studies with glypican-3 (GPC-3)-directed vaccines [47]; clearly, further comprehensive analyses are warranted to strengthen these preliminary data. DCs have an important role in antigen presentation to Tcells in HCC, enhancing immune response; therefore, the use of DC vaccines has been tested in a Phase II trial showing promising benefit [48]. The use of viruses with high tropism to HCC cancer cells has been investigated in the HCC field as adenoviruses, vesicular stomatitis virus (VSV), vaccinia virus (VV), and Herpes Simplex Virus (HSV) [49,50]. To enhance anti-tumor efficacy, the virus genome is engineered with several genes that confer enhanced immunity $[51,52]$. Several preclinical data have provided encouraging results and some clinical trials with adenoviruses and vaccinia viruses modified with the deletion of TK and VGF and insertion of human GM-CSF have been conducted, showing some signs of activity, but the tolerability profile of these novel approaches needs to be confirmed in larger trials, including combination with approved ICIs [53].

Giving impressive results obtained with CAR-T-cell therapy in hematological malignancies, several preclinical trials have been conducted in solid tumors [54]. In HCC there are many obstacles to be removed such as the immunosuppressive microenvironment and the antigen heterogeneity that limits CAR-T-cell treatment efficacy, and preclinical and clinical studies are currently being conducted to further investigate this innovative strategy that represents a promising option for patients with HCC [55].

\section{Correct Treatment Sequence after Implementation of ICI in the HCC Therapeutic Landscape}

After the outstanding results of the IMbrave trial, atezolizumab plus bevacizumab combination has been considered to be the optimal strategy for first-line treatment. An important challenge is represented by the choice of the correct sequence for the treatment of later lines of therapy, as described in Figure 1. First, atezolizumab plus bevacizumab is the first treatment option for advanced disease, and sorafenib and lenvatinib MKIs, which have represented for years the only choice for first-line treatment, can now be considered for subsequent lines after atezolizumab bevacizumab failure. However, SHARP and REFLECT trials have investigated the use of sorafenib and lenvatinib in first-line treatment and, so far, only retrospective analyses of their use in second-line treatment have been published. Moreover, a limitation in the use of atezolizumab could be determined by the presence of uncontrolled autoimmune disease, while bevacizumab should be avoided in patients with high risk of bleeding. The other approved MKIs that are used in second-line treatment (regorafenib, ramucirumab, cabozantinib) have been investigated after sorafenib failure and there is a lack of evidence of their efficacy after atezolizumab plus bevacizumab failure. On the other hand, as the efficacy of the abovementioned MKIs has been largely demonstrated but there is no evidence of their direct comparison, international guidelines suggest their use even after atezolizumab plus bevacizumab progression. Another still-unanswered question is if an anti-PD-1 agent (nivolumab or pembrolizumab) could still be offered after progression of ICI-based therapy, as the anti-PD-L1 atezolizumab. In the same way, is it possible to treat patients with an anti-VEGF (ramucirumab) after bevacizumab failure? This 
strategy has proven effective in other diseases such as colorectal cancer but in HCC, data are very limited. Furthermore, so far only cabozantinib has been approved for third-line treatment, as $27 \%$ of patients enrolled in the CELESTIAL trial had already received two precedent treatment lines (sorafenib in the first line, ICI in the second). Nevertheless, there is no evidence of cabozantinib treatment after sorafenib and regorafenib or sorafenib and ramucirumab drugs. This and other questions remain unsolved. Future large clinical trials that include different treatment scenarios with combinations of different classes of agents in advanced settings could help define the correct treatment sequence of HCC.

\section{Conclusions and Future Perspectives}

HCC is a complex disease characterized by relevant immune-escape mechanisms that might be solved with immune strategies. However, the implementation of ICIs in the algorithm of HCC treatment has experienced a change in recent years as Phase III confirmatory clinical trials of pembrolizumab and nivolumab in second-line treatment unfortunately failed to reach the primary endpoint and updates regarding the accelerated approval by regulatory agencies are strongly awaited. Moreover, the use of ICIs in combination with different strategies such as target agents or locoregional treatments (TACE, SIRT) seems to provide better efficacy results, although safety matters such as hepatotoxicity still need to be addressed. Thus, identifying the correct sequence treatment and finding the "right place" for immunotherapy in the therapeutic armamentarium of patients with HCC represents a challenge. Furthermore, principal target agents and ICIs are still reserved for a subset of patients with HCC retaining a good performance status (Child-Pugh A, ECOG 0-1), a condition difficult to achieve most of all in second-line and subsequent lines of treatment and that sometimes could change during treatment. So far, we only have limited data of patients with Child-Pugh B with sorafenib and nivolumab. Therefore, analyses regarding real-world experience with ICI in HCC treatment could provide more comprehensive data of a heterogeneous population, to be taken into consideration to further extend the current indication of immune treatment in advanced HCC. Even though HCC is one of the principal causes of death worldwide, it is a complicated dynamic disease in which the immune system retains a strong role, and comprehensive knowledge is presently limited as immune resistance mechanisms are still unknown. In fact, the RR of patients that receive immunotherapy remains poor, and biomarker-guided strategies are strongly requested to increase the number of patients that could respond to treatment. To this extent, only ramucirumab represents so far a biomarker-guided treatment, while prognostic and predictive immune biomarkers are still unknown. PD-L1 expression is a well-recognized predictive biomarker for different solid tumors, but in HCC was assessed only in a retrospective analysis from the CheckMate 040 trial, in which no association was found with efficacy outcomes [12]. In this scenario, new data from preclinical trials, such as in vitro drug screening followed by validation in in vivo models, implementation into the immune therapeutic armamentarium of innovative tools such as liquid biopsy and genomic and transcriptomic signatures (Figure 2) that identify poor responders to immunotherapies could provide a "precision medicine"-based approach in the near future. 


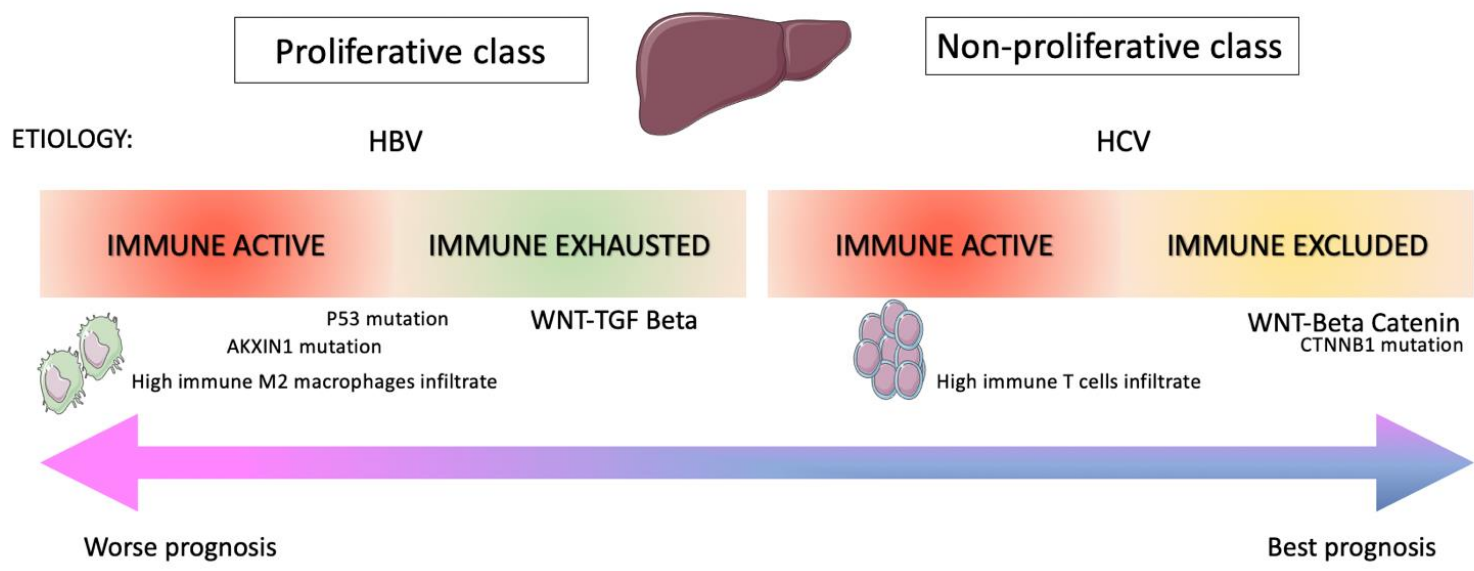

Figure 2. Molecular features of HCC immune classes.

Author Contributions: Conceptualization, G.M. and E.M.; methodology, G.M., D.C. and E.M.; writing—original draft preparation, G.M.; writing—review and editing G.M., S.N., C.M.D.C.; supervision, F.P., V.N., W.S., F.U., M.S., M.N., M.D., A.F., F.S., C.M.D.C., S.N., F.C. and E.M. All authors have read and agreed to the published version of the manuscript.

Funding: This research received no external funding.

Conflicts of Interest: D.C. received a travel support from Sanofi. F.C. has served as advisor and speaker for Roche, Amgen, Merck-Serono, Pfizer, Sanofi, Bayer, Servier, BMS, Cellgene, Lilly. Received institutional Research Grants form Bayer, Roche, Merck-Serono, Amgen, AstraZeneca, Takeda. E.M. has served as advisor and speaker for Astra Zeneca, Amgen, Bayer, Merck-Serono, Roche, Sanofi, Servier, Pierre Fabre. G.M., F.P., V.N., W.S., F.U., M.S., M.N., M.D., A.F., F.S., C.M.D.C., S.N. declare no competing interests.

\section{References}

1. Bray, F.; Ferlay, J.; Soerjomataram, I.; Siegel, R.L.; Torre, L.A.; Jemal, A. Global cancer statistics 2018: GLOBOCAN estimates of incidence and mortality worldwide for 36 cancers in 185 countries. CA Cancer J. Clin. 2018, 68, 394-424. [CrossRef]

2. Cariani, E.; Missale, G. Immune landscape of hepatocellular carcinoma microenvironment: Implications for prognosis and therapeutic applications. Liver Int. 2019, 39, 1608-1621. [CrossRef]

3. Llovet, J.M.; Brú, C.; Bruix, J. Prognosis of Hepatocellular Carcinoma: The BCLC Staging Classification. Semin. Liver Dis. 1999, 19, 329-338. [CrossRef] [PubMed]

4. Vogel, A.; Saborowski, A. Current strategies for the treatment of intermediate and advanced hepatocellular carcinoma. Cancer Treat. Rev. 2020, 82, 101946. [CrossRef] [PubMed]

5. Erstad, D.J.; Tanabe, K.K. Hepatocellular carcinoma: Early-stage management challenges. J. Hepatocell. Carcinoma 2017, 4, 81-92. [CrossRef] [PubMed]

6. $\quad$ Llovet, J.M.; Ricci, S.; Mazzaferro, V.; Hilgard, P.; Gane, E.; Blanc, J.F.; De Oliveira, A.C.; Santoro, A.; Raoul, J.L.; Forner, A.; et al. Sorafenib in Advanced Hepatocellular Carcinoma. N. Engl. J. Med. 2008, 359, 378-390. [CrossRef]

7. Tsilimigras, D.I.; Bagante, F.; Sahara, K.; Moris, D.; Hyer, J.M.; Wu, L.; Ratti, F.; Marques, H.P.; Soubrane, O.; Paredes, A.Z.; et al. Prognosis after Resection of Barcelona Clinic Liver Cancer (BCLC) Stage 0, A, and B Hepatocellular Carcinoma: A Comprehensive Assessment of the Current BCLC Classification. Ann. Surg. Oncol. 2019, 26, 3693-3700. [CrossRef]

8. Kudo, M.; Finn, R.S.; Qin, S.; Han, K.-H.; Ikeda, K.; Piscaglia, F.; Baron, A.; Park, J.-W.; Han, G.; Jassem, J.; et al. Lenvatinib versus sorafenib in first-line treatment of patients with unresectable hepatocellular carcinoma: A randomised phase 3 non-inferiority trial. Lancet 2018, 391, 1163-1173. [CrossRef]

9. Bruix, J.; Qin, S.; Merle, P.; Granito, A.; Huang, Y.-H.; Bodoky, G.; Pracht, M.; Yokosuka, O.; Rosmorduc, O.; Breder, V.; et al. Regorafenib for patients with hepatocellular carcinoma who progressed on sorafenib treatment (RESORCE): A randomised, double-blind, placebo-controlled, phase 3 trial. Lancet 2017, 389, 56-66. [CrossRef]

10. Abou-Alfa, G.K.; Meyer, T.; Cheng, A.-L.; El-Khoueiry, A.B.; Rimassa, L.; Ryoo, B.-Y.; Cicin, I.; Merle, P.; Chen, Y.; Park, J.-W.; et al. Cabozantinib in Patients with Advanced and Progressing Hepatocellular Carcinoma. N. Engl. J. Med. 2018, 379, 54-63. [CrossRef]

11. Zhu, A.X.; Kang, Y.-K.; Yen, C.-J.; Finn, R.S.; Galle, P.R.; Llovet, J.M.; Assenat, E.; Brandi, G.; Pracht, M.; Lim, H.Y.; et al Ramucirumab after sorafenib in patients with advanced hepatocellular carcinoma and increased $\alpha$-fetoprotein concentrations (REACH-2): A randomised, double-blind, placebo-controlled, phase 3 trial. Lancet Oncol. 2019, 20, 282-296. [CrossRef] 
12. El-Khoueiry, A.B.; Sangro, B.; Yau, T.; Crocenzi, T.S.; Kudo, M.; Hsu, C.; Kim, T.-Y.; Choo, S.-P.; Trojan, J.; Welling, T.H.; et al. Nivolumab in patients with advanced hepatocellular carcinoma (CheckMate 040): An open-label, non-comparative, phase $1 / 2$ dose escalation and expansion trial. Lancet 2017, 389, 2492-2502. [CrossRef]

13. Zhu, A.X.; Finn, R.S.; Edeline, J.; Cattan, S.; Ogasawara, S.; Palmer, D.; Verslype, C.; Zagonel, V.; Fartoux, L.; Vogel, A.; et al. Pembrolizumab in patients with advanced hepatocellular carcinoma previously treated with sorafenib (KEYNOTE-224): A non-randomised, open-label phase 2 trial. Lancet Oncol. 2018, 19, 940-952. [CrossRef]

14. Finn, R.S.; Qin, S.; Ikeda, M.; Galle, P.R.; Ducreux, M.; Kim, T.-Y.; Kudo, M.; Breder, V.; Merle, P.; Kaseb, A.O. Atezolizumab plus Bevacizumab in Unresectable Hepatocellular Carcinoma. N. Engl. J. Med. 2020, 382, 1894-1905. [CrossRef] [PubMed]

15. Nishida, N.; Kudo, M. Immunological Microenvironment of Hepatocellular Carcinoma and Its Clinical Implication. Oncology 2017, 92, 40-49. [CrossRef]

16. Crispe, I.N. Hepatic T cells and liver tolerance. Nat. Rev. Immunol. 2003, 3, 51-62. [CrossRef] [PubMed]

17. Liu, Z.; Zhang, Y.; Shi, C.; Zhou, X.; Xu, K.; Jiao, D.; Sun, Z.; Han, X. A novel immune classification reveals distinct immune escape mechanism and genomic alterations: Implications for immunotherapy in hepatocellular carcinoma. J. Transl. Med. 2021, 19, 5. [CrossRef] [PubMed]

18. Fu, Y.; Liu, S.; Zeng, S.; Shen, H. From bench to bed: The tumor immune microenvironment and current immunotherapeutic strategies for hepatocellular carcinoma. J. Exp. Clin. Cancer Res. 2019, 38, 396. [CrossRef] [PubMed]

19. Kurebayashi, Y.; Ojima, H.; Tsujikawa, H.; Kubota, N.; Maehara, J.; Abe, Y.; Kitago, M.; Shinoda, M.; Kitagawa, Y.; Sakamoto, M. Landscape of immune microenvironment in hepatocellular carcinoma and its additional impact on histological and molecular classification. Hepatology 2018, 68, 1025-1041. [CrossRef] [PubMed]

20. Kakumu, S.; Ito, S.; Ishikawa, T.; Mita, Y.; Tagaya, T.; Fukuzawa, Y.; Yoshioka, K. Decreased function of peripheral blood dendritic cells in patients with hepatocellular carcinoma with hepatitis B and C virus infection. J. Gastroenterol. Hepatol. 2000, 15, 431-436. [CrossRef]

21. Chen, J.; Gingold, J.A.; Su, X. Immunomodulatory TGF- $\beta$ Signaling in Hepatocellular Carcinoma. Trends Mol. Med. 2019, 25, 1010-1023. [CrossRef]

22. Horst, A.K.; Kumashie, K.G.; Neumann, K.; Diehl, L.; Tiegs, G. Antigen presentation, autoantibody production, and therapeutic targets in autoimmune liver disease. Cell. Mol. Immunol. 2021, 18, 92-111. [CrossRef]

23. Bai, J.; Liang, P.; Li, Q.; Feng, R.; Liu, J. Cancer Immunotherapy-Immune Checkpoint Inhibitors in Hepatocellular Carcinoma. Recent Patents Anti-Cancer Drug Discov. 2021, 16, 1. [CrossRef]

24. He, S.; Jiang, W.; Fan, K.; Wang, X. The Efficacy and Safety of Programmed Death-1 and Programmed Death Ligand 1 Inhibitors for the Treatment of Hepatocellular Carcinoma: A Systematic Review and Meta-Analysis. Front. Oncol. 2021, 11, 881. [CrossRef]

25. Federico, P.; Petrillo, A.; Giordano, P.; Bosso, D.; Fabbrocini, A.; Ottaviano, M.; Rosanova, M.; Silvestri, A.; Tufo, A.; Cozzolino, A.; et al. Immune Checkpoint Inhibitors in Hepatocellular Carcinoma: Current Status and Novel Perspectives. Cancers 2020, $12,3025$. [CrossRef]

26. Yau, T.; Park, J.W.; Finn, R.S.; Cheng, A.L.; Mathurin, P.; Edeline, J.; Kudo, M.; Han, K.H.; Harding, J.J.; Merle, P.; et al. CheckMate 459: A randomized, multi-center phase III study of nivolumab (NIVO) vs sorafenib (SOR) as first-line (1L) treatment in patients (pts) with advanced hepatocellular carcinoma (aHCC). Ann. Oncol. 2019, 30, v874-v875. [CrossRef]

27. Finn, R.S.; Ryoo, B.-Y.; Merle, P.; Kudo, M.; Bouattour, M.; Lim, H.-Y.; Breder, V.V.; Edeline, J.; Chao, Y.; Ogasawara, S.; et al. Results of KEYNOTE-240: Phase 3 study of pembrolizumab (Pembro) vs best supportive care (BSC) for second line therapy in advanced hepatocellular carcinoma (HCC). J. Clin. Oncol. 2019, 37, 4004. [CrossRef]

28. Lee, M.S.; Ryoo, B.-Y.; Hsu, C.-H.; Numata, K.; Stein, S.; Verret, W.; Hack, S.P.; Spahn, J.; Liu, B.; Abdullah, H.; et al. Atezolizumab with or without bevacizumab in unresectable hepatocellular carcinoma (GO30140): An open-label, multicentre, phase 1b study. Lancet Oncol. 2020, 21, 808-820. [CrossRef]

29. Finn, R.S.; Qin, S.; Ikeda, M.; Galle, P.R.; Ducreux, M.; Kim, T.-Y.; Lim, H.Y.; Kudo, M.; Breder, V.V.; Merle, P.; et al. IMbrave150: Updated overall survival (OS) data from a global, randomized, open-label phase III study of atezolizumab (atezo) + bevacizumab (bev) versus sorafenib (sor) in patients (pts) with unresectable hepatocellular carcinoma (HCC). J. Clin. Oncol. 2021, $39,267$. [CrossRef]

30. Wei, S.C.; Duffy, C.R.; Allison, J.P. Fundamental Mechanisms of Immune Checkpoint Blockade Therapy. Cancer Discov. 2018, 8, 1069-1086. [CrossRef] [PubMed]

31. Larkin, J.; Chiarion-Sileni, V.; Gonzalez, R.; Grob, J.-J.; Rutkowski, P.; Lao, C.D.; Cowey, C.L.; Schadendorf, D.; Wagstaff, J.; Dummer, R.; et al. Five-Year Survival with Combined Nivolumab and Ipilimumab in Advanced Melanoma. N. Engl. J. Med. 2019, 381, 1535-1546. [CrossRef]

32. Hellmann, M.D.; Paz-Ares, L.; Caro, R.B.; Zurawski, B.; Kim, S.-W.; Costa, E.C.; Park, K.; Alexandru, A.; Lupinacci, L.; De la Mora, J.E.; et al. Nivolumab plus ipilimumab in advanced non-small-cell lung cancer. N. Engl. J. Med. 2019, 381, 2020-2031. [CrossRef]

33. Sangro, B.; Gomez-Martin, C.; de la Mata, M.; Iñarrairaegui, M.; Garralda, E.; Barrera, P.; Riezu-Boj, J.-I.; Larrea, E.; Alfaro, C.; Sarobe, P.; et al. A clinical trial of CTLA-4 blockade with tremelimumab in patients with hepatocellular carcinoma and chronic hepatitis C. J. Hepatol. 2013, 59, 81-88. [CrossRef]

34. Kelley, R.K.; Abou-Alfa, G.K.; Bendell, J.C.; Kim, T.-Y.; Borad, M.J.; Yong, W.-P.; Morse, M.; Kang, Y.; Rebelatto, M.; Makowsky, M.; et al. Phase I/II study of durvalumab and tremelimumab in patients with unresectable hepatocellular carcinoma (HCC): Phase I safety and efficacy analyses. J. Clin. Oncol. 2017, 35, 4073. [CrossRef] 
35. Kelley, R.K.; Sangro, B.; Harris, W.; Ikeda, M.; Okusaka, T.; Kang, Y.-K.; Qin, S.; Tai, D.W.-M.; Lim, H.Y.; Yau, T.; et al. Safety, Efficacy, and Pharmacodynamics of Tremelimumab Plus Durvalumab for Patients With Unresectable Hepatocellular Carcinoma: Randomized Expansion of a Phase I/II Study. J. Clin. Oncol. 2021, 39, 2991-3001. [CrossRef]

36. Abou-Alfa, G.K.; Chan, S.L.; Furuse, J.; Galle, P.R.; Kelley, R.K.; Qin, S.; Armstrong, J.; Darilay, A.; Vlahovic, G.; Negro, A.; et al. A randomized, multicenter phase 3 study of durvalumab (D) and tremelimumab (T) as first-line treatment in patients with unresectable hepatocellular carcinoma (HCC): HIMALAYA study. J. Clin. Oncol. 2018, 36, TPS4144. [CrossRef]

37. Morse, M.A.; Sun, W.; Kim, R.; He, A.R.; Abada, P.B.; Mynderse, M.; Finn, R.S. The Role of Angiogenesis in Hepatocellular Carcinoma. Clin. Cancer Res. 2019, 25, 912-920. [CrossRef]

38. Zhu, A.X.; Park, J.O.; Ryoo, B.-Y.; Yen, C.-J.; Poon, R.; Pastorelli, D.; Blanc, J.-F.; Chung, H.; Baron, A.D.; Pfiffer, T.E.F.; et al. Ramucirumab versus placebo as second-line treatment in patients with advanced hepatocellular carcinoma following first-line therapy with sorafenib (REACH): A randomised, double-blind, multicentre, phase 3 trial. Lancet Oncol. 2015, 16, 859-870. [CrossRef]

39. Evoron, T.; Emarcheteau, E.; Epernot, S.; Ecolussi, O.; Etartour, E.; Etaieb, J.; Eterme, M. Control of the Immune Response by Pro-Angiogenic Factors. Front. Oncol. 2014, 4, 70. [CrossRef]

40. Lin, C.-C.; Golan, T.; Corral, J.; Moreno, V.; Chung, H.; Wasserstrom, H.; Yang, J.; Mi, G.; Bang, Y.-J. Phase 1 study of ramucirumab (R) plus durvalumab (D) in patients (pts) with locally advanced and unresectable or metastatic gastrointestinal or thoracic malignancies (NCT02572687); Phase 1a results. Ann. Oncol. 2016, 27, viii1. [CrossRef]

41. Finn, R.S.; Ikeda, M.; Zhu, A.X.; Sung, M.W.; Baron, A.D.; Kudo, M.; Okusaka, T.; Kobayashi, M.; Kumada, H.; Kaneko, S.; et al. Phase $\mathrm{Ib}$ Study of Lenvatinib Plus Pembrolizumab in Patients With Unresectable Hepatocellular Carcinoma. J. Clin. Oncol. 2020, 38, 2960-2970. [CrossRef] [PubMed]

42. Xu, J.; Shen, J.; Gu, S.; Zhang, Y.; Wu, L.; Wu, J.; Shao, G.; Zhang, Y.; Xu, L.; Yin, T.; et al. Camrelizumab in Combination with Apatinib in Patients with Advanced Hepatocellular Carcinoma (RESCUE): A Nonrandomized, Open-label, Phase II Trial. Clin. Cancer Res. 2021, 27, 1003-1011. [CrossRef]

43. Zhang, Y.; Xu, J.; Shen, J.; Gu, S.; Wu, L.; Wu, J.; Shao, G.; Zhang, Y.; Xu, L.; Yin, T.; et al. Update on overall survival (OS) of RESCUE: An open-label, phase 2 trial of camrelizumab (C) in combination with apatinib (A) in patients with advanced hepatocellular carcinoma (HCC). J. Clin. Oncol. 2021, 39, 4076. [CrossRef]

44. Kudo, M.; Ueshima, K.; Ikeda, M.; Torimura, T.; Tanabe, N.; Aikata, H.; Izumi, N.; Yamasaki, T.; Nojiri, S.; Hino, K.; et al. Randomised, multicentre prospective trial of transarterial chemoembolisation (TACE) plus sorafenib as compared with TACE alone in patients with hepatocellular carcinoma: TACTICS trial. Gut 2020, 69, 1492-1501. [CrossRef] [PubMed]

45. Pinato, D.; Cole, T.; Bengsch, B.; Tait, P.; Sayed, A.; Abomeli, F.; Gramenitskaya, D.; Allara, E.; Thomas, R.; Ward, C.; et al. A phase Ib study of pembrolizumab following trans-arterial chemoembolization (TACE) in hepatocellular carcinoma (HCC): PETAL. Ann. Oncol. 2019, 30, v288. [CrossRef]

46. Alisa, A.; Ives, A.; Pathan, A.A.; Navarrete, C.V.; Williams, R.; Bertoletti, A.; Behboudi, S. Analysis of CD4+ T-Cell Responses to a Novel $\alpha$-Fetoprotein-Derived Epitope in Hepatocellular Carcinoma Patients. Clin. Cancer Res. 2005, 11, 6686-6694. [CrossRef]

47. Sawada, Y.; Yoshikawa, T.; Nobuoka, D.; Shirakawa, H.; Kuronuma, T.; Motomura, Y.; Mizuno, S.; Ishii, H.; Nakachi, K.; Konishi, M.; et al. Phase I Trial of a Glypican-3-Derived Peptide Vaccine for Advanced Hepatocellular Carcinoma: Immunologic Evidence and Potential for Improving Overall Survival. Clin. Cancer Res. 2012, 18, 3686-3696. [CrossRef]

48. Palmer, D.H.; Midgley, R.S.; Mirza, N.; Torr, E.E.; Ahmed, F.; Steele, J.C.; Steven, N.M.; Kerr, D.J.; Young, L.S.; Adams, D.H. A phase II study of adoptive immunotherapy using dendritic cells pulsed with tumor lysate in patients with hepatocellular carcinoma. Hepatology 2009, 49, 124-132. [CrossRef] [PubMed]

49. Heo, J.; Breitbach, C.J.; Moon, A.; Kim, C.W.; Patt, R.; Kim, M.K.; Lee, Y.K.; Oh, S.Y.; Woo, H.Y.; Parato, K.; et al. Sequential Therapy With JX-594, A Targeted Oncolytic Poxvirus, Followed by Sorafenib in Hepatocellular Carcinoma: Preclinical and Clinical Demonstration of Combination Efficacy. Mol. Ther. 2011, 19, 1170-1179. [CrossRef]

50. Ady, J.W.; Heffner, J.; Mojica, K.; Johnsen, C.; Belin, L.J.; Love, D.; Chen, C.-T.; Pugalenthi, A.; Klein, E.; Chen, N.G.; et al. Oncolytic immunotherapy using recombinant vaccinia virus GLV-1h68 kills sorafenib-resistant hepatocellular carcinoma efficiently. Surgery 2014, 156, 263-269. [CrossRef] [PubMed]

51. Altomonte, J.; Marozin, S.; Schmid, R.M.; Ebert, O. Engineered Newcastle Disease Virus as an Improved Oncolytic Agent Against Hepatocellular Carcinoma. Mol. Ther. 2010, 18, 275-284. [CrossRef] [PubMed]

52. Yoo, S.Y.; Badrinath, N.; Woo, H.Y.; Heo, J. Oncolytic Virus-Based Immunotherapies for Hepatocellular Carcinoma. Mediat. Inflamm. 2017, 2017, 5198798. [CrossRef]

53. Park, B.-H.; Hwang, T.; Liu, T.-C.; Sze, D.Y.; Kim, J.-S.; Kwon, H.-C.; Oh, S.Y.; Han, S.-Y.; Yoon, J.-H.; Hong, S.-H.; et al. Use of a targeted oncolytic poxvirus, JX-594, in patients with refractory primary or metastatic liver cancer: A phase I trial. Lancet Oncol. 2008, 9, 533-542. [CrossRef]

54. Efficace, F.; Vignetti, M. Quality of life and CAR-T cell therapy in children, adolescents, and young adults with haematological malignancies. Lancet Oncol. 2019, 20, 1625-1626. [CrossRef]

55. Gao, H.; Li, K.; Tu, H.; Pan, X.; Jiang, H.; Shi, B.; Kong, J.; Wang, H.; Yang, S.; Gu, J.; et al. Development of T Cells Redirected to Glypican-3 for the Treatment of Hepatocellular Carcinoma. Clin. Cancer Res. 2014, 20, 6418-6428. [CrossRef] [PubMed] 